\title{
F.H. Bradley: ¿un espinosista hegeliano?
}

\section{F.H. Bradley: An Hegelian Spinozist?}

RESUMEN: La doctrina de Spinoza constituye una de las principales inspiraciones del más célebre entre los idealistas británicos, F.H. Bradley, cuya filosofía se caracteriza a su vez por elementos típicos del idealismo alemán y, en particular, de Hegel. Según A. Seth PringlePattison, la presencia de estas dos tendencias conflictivas reduce el planteamiento de Appearance and Reality a una disposición filosófica esencialmente mística. Sin embargo, a pesar de las reales influencias de Spinoza y Hegel en el contexto del idealismo británico, los cimientos de la metafísica bradleyana (la incognoscibilidad de lo Absoluto, su transcendencia respecto al pensamiento, el rechazo del panteísmo y del panlogismo) sugieren una postura difícilmente identificable con Spinoza o Hegel, o con ambos.

PALABRAS CLAVE: ABSOLUTO - IDEALISMO BRITÁNICO - MONISMO - PANLOGISMO - PANTEÍSMO.

\begin{abstract}
Spinoza's doctrine represents one of the greatest inspirations for the most well-known representative of the British Idealism, F.H. Bradley, whose philosophy is also marked by some of the most typical elements of German Idealism, especially those of Hegel. According to Seth Pringle-Pattison, the presence of these conflictive tendencies reduce the standpoint of Appearance and Reality to an essentially mystic philosophical attitude. However, despite the real influences of Spinoza and Hegel in the context of British Idealism, the foundations of Bradley's metaphysics (the unknowability of the Absolute and its transcendence, as well as the rejection of pantheism and panlogism) suggest that Bradley is defending a perspective that can hardly be identified with Spinoza or Hegel, or with both of them.
\end{abstract}

KEY WORDS: ABSOLUTE - BRITISH IDEALISM - MONISM - PANLOGISM PANTHEISM. 


\section{INTRODUCCIÓN}

Como es conocido, Francis Herbert Bradley (1846-1924) es considerado 1 por todos los críticos el intérprete más importante del idealismo británico, un movimiento tanto interesante cuanto difícilmente interpretable que domina la escena filosófica en Gran Bretaña durante todo el siglo xIx y en las primeras décadas del siglo $\mathrm{xx}^{1}{ }^{1}$ La historia filosófica y hermenéutica del movimiento depende en gran parte de las interpretaciones realizadas sobre Kant y Hegel, y, posteriormente, de la lectura anti-idealista de George Edward Moore (18731958) y Bertrand Russell (1872-1970). Un estudio recién publicado recuerda que «la crónica de la filosofía británica como se enseña en nuestras universidades acaba con Mill y empieza otra vez con Russell y Moore, como si no hubiese pasado nada en el período intermedio. Pero, al intentar rellenar este vacío, nos quedamos sorprendidos».2

Este sentimiento de sorpresa se debe al número casi infinito de temas y argumentos desarrollados y defendidos por los filósofos idealistas, incluso en los años anteriores a la generación más prolífica y relevante de Bradley, Bernard Bosanquet (1848-1923) y John Ellis McTaggart (1866-1925). ${ }^{3}$ Bien mirado, el fenómeno no debería causar asombro, pues ese periodo intermedio dura casi un siglo y ha sido protagonizado por figuras del calibre de Thomas Carlyle (1795-1881), los hermanos John y Edward Caird (1820-1898 y 1835-1908), Samuel Taylor Coleridge (1772-1834), James Frederick Ferrier (1808-1864), Thomas Hill

[1] Véanse G. BedelL, «Bradley’s Monistic Idealism», The Thomist: A Speculative Quarterly Review, 34, 4 (Oct 1, 1970), pp. 568-583; H. HøfFding, Modern Philosophers, London: MacMillan and Co., 1915; R. Metz, A Hundred Years of British Philosophy, London: George Allen \& Unwin ltd., 1938; J. Pucelle, L'idéalisme en Angleterre de Coleridge à Bradley, Neuchatel: La Bacconière, 1955; G. Watts Cunningham, The Idealistic Argument in Recent British and American Philosophy, New York: Books for Libraries Press, 1967.

[2] W.J. Mander, British Idealism. A History, Oxford: Oxford University Press, 2011, p. 1. Todas las traducciones del inglés son del autor de este texto.

[3] Es decir, cuando la falta de abertura hacia el continente impedía todavía un masivo intercambio de conocimientos con los filósofos contemporáneos. Véanse M. CaRbó I RiBUGENT, «Henry Crabb Robinson i el context filosòfic de la primera importació de Kant a Anglaterra», Anuari de la Societat Catalana de Filosofia, XIx (2008), pp. 35-56; G. MicheLI, «The early reception of Kant's Thought in England 1785-1805», en T. McWalter-G. MacDonald Ross (edited by), Kant and his influence, London: Thoemmes Press, 1990, pp. 202-314; J.H. MuIrHEAD, «How Hegel came to England», Mind New Series, Vol. 36, No. 144 (Oct., 1927), pp. 423-447; A. Seth-R.B. Haldane (edited by), Essays in Philosphical Criticism, with a Preface of E. Caird, New York: Burt Franklin, 1971; R. Wellek, Immanuel Kant in England, 1793-1838, Princeton University Press, 1931. 
Green (1836-1882), Harold Henry Joachim (1868-1938), John Henry Muirhead (1855-1940), Hastings Rashdall (1858-1924), James Hutchison Stirling (18201909), William Wallace (1844-1897), William Wordsworth (1770-1850).

Entre los ingredientes que concurren a influenciar y enriquecer la producción metafísica y filosófica en el Reino Unido, así como a favorecer la aportación de ideas nuevas, cabe recordar - además de las influencias de Kant y Hegel - la Spinoza Renaissance, cuya relevancia para el idealismo, como demuestra Robinson, depende de su papel fundamental para la comprensión de la filosofía de Schelling. ${ }^{4}$ Asimismo, además de los protagonistas que se acaban de mencionar, resulta excepcionalmente interesante recordar la figura de Andrew Seth Pringle-Pattison (1856-1931): ${ }^{5}$ su crítica a Appearance and Reality, que Bradley publica en 1893, tiene el objetivo de mostrar que la síntesis de los elementos hegelianos y spinozianos, reconocibles en toda su filosofía, y que lleva a cabo sin apoyarse en el método dialéctico, refleja un planteamiento místico insuficiente para construir un sistema de lo Absoluto.

El artículo se desarrollará por tanto de la siguiente manera: en la sección II trataré de esbozar la doctrina metafísica de Bradley recordando sus elementos principales. A continuación, en el párrafo III, expondré los argumentos del ensayo «A New Theory of the Absolute. Mr. Bradley's Appearance and Reality», en el que Seth Pringle-Pattison, como se ha mencionado, hace hincapié en la presencia de las tendencias spinozista y hegeliana a la hora de discutir la filosofía de Bradley. ${ }^{6}$ Para concluir, en la sección IV mostraré como la lectura propuesta en el artículo no resulta satisfactoria, ya que, a pesar de la confluencia de elementos de las filosofías de Spinoza y Hegel, ninguno de ellos lleva a coincidir con el planteamiento de Bradley.

\section{APPEARANCE AND REALITY: CONSIDERACIONES PRELIMINARES}

La obra justifica indudablemente la fama y la relevancia de su autor en el marco del pensamiento británico en particular e idealista en general. Su

[4] Para consideraciones ulteriores o de carácter histórico, remito a M. CARbó I RIBUgENT, loc. cit; A. Deregibus, «Bradley e Spinoza. L'iper-spinozismo di F.H. Bradley», Giornale di Metafisica Nuova Serie, x (1988), pp. 339-392 y xi (1989), pp. 3-40; S. Newlands, «More Recent Idealist Readings of Spinoza», Philosophy Compass, 6/2 (2011), pp. 109-119.

[5] Andrew Seth Pringle-Pattison nació Andrew Seth y publicó con este nombre hasta 1898, cuando, con el fin de obtener una herencia, añadió a su nombre el de la familia Pringle-Pattison. Por esta razón, en la nota 3 me he referido al autor solamente con su primer apellido.

[6] El ensayo está publicado en A. Seth Pringle-Pattison, Man's Place in Cosmos and Other Essays, Edinburgh-London: Blackwood and Sons, 1897, pp. 129-225.

[7] El título original del texto es F.H. Bradley, Appearance and Reality: A Metaphysical Essay. Citaré aquí la edición de Elibron Classics, 2005 (impresión anastática de F.H. BRAdLeY, Appearance and Reality: A Metaphysical Essay, London: Swan Sonnenschein \& Co., 1893). 
aspecto más destacado consiste sin duda en la esencia cuasi contradictoria de los dos libros en los que se desarrolla su pensamiento. El primero de ellos, en efecto, es un análisis escéptico de las categorías lógico-ontológicas que definen y constituyen el mundo fenoménico. La incomprensibilidad de las relaciones, un argumento que se distingue por poco de una verdadera paradoja, justifica el rechazo de las nociones de espacio, tiempo, movimiento, causa, actividad, Yo, cosa: de manera más general, de la independencia de todo contenido finito respecto a la Realidad considerada $a b$ integro. ${ }^{8}$ La naturaleza contradictoria de esta esfera, según demuestra la famosa doctrina de la distinción entre contenido (what) y existencia (that), condenaría la ontología al simple status de apariencia y pondría al conocimiento frente a un categórico y difícil aut-aut: la renuncia definitiva a la verdad o su propio suicidio. ${ }^{9}$

En el segundo libro, Bradley complementa la deriva escéptica con una pars costruens de carácter metafísico y monista, gracias al soporte de los argumentos desarrollados en The Principles of Logic, publicada en $1883:{ }^{10}$ para concebir lo Absoluto es necesario comprender que «se da un solo sujeto sobre el que se pueda hacer una aserción verdadera y objetivamente válida y que este sujeto no es otra cosa sino la Realidad misma». ${ }^{11}$ En efecto, como observa correctamente Cassirer, para Bradley solo «desde la efectividad como totalidad [als Ganzem], no desde sus partes singulares, es posible formular un juicio válido, un juicio capaz de reivindicar un derecho a la certeza metafísica». ${ }^{12}$ Lo Absoluto debe de poder conciliar y superar las contradicciones de los fenómenos y de la esfera

[8] En palabras de Bradley: «toda forma relacional de pensamiento - a saber, toda forma que procede mediante un esquema que se basa en términos y relaciones - debe darnos la apariencia y no la realidad. Se trata solamente de un expediente, un recurso, un compromiso puramente práctico, y en cuanto tal indiscutiblemente necesario, mas, en última instancia, indefendible» [F.H. Bradley, op. cit., p. 33]. Véanse S. Candlish, «The Truth About F.H. Bradley», Mind New Series, Vol. 98, No. 391 (Jul., 1989), pp. 331-348; M. Truglia, F.H. Bradley e la genesi della filosofia analitica. Contributi per una definizione, Milano: Unicopli, 2007.

[9] Véanse G. Bertolotti, Le stagioni dellassoluto: saggio su Bradley, Firenze: La Nuova Italia, 1995; M. CARdani, «Por un puñado de... táleros. Existencia y contenido empírico en F. H. Bradley», Comprendre: Revista catalana de filosofia, Vol. 16, Núm. 2 (2014), pp. 51-70; T. S. Eliot, Knowledge and Experience in the Philosophy of F. H. Bradley, London: Faber \& Faber, 1964; R. Wollheim, F.H. Bradley, Harmondsworth-Baltimore-Ringwood: Penguin, 1969.

[10] Véase F.H. Bradley, The Principles of Logic, Second Edition revised with Commentary and Terminal Essays, edited by H. Milford, 2 Vols., London: Oxford University Press, 1950.

[11] E. Cassirer, Erkenntnis, Begriff, Kultur, herausgegeben, eingeleitet sowie mit Anmerkungen und Registern versehen von R.A. Bast, Hamburg: Meiner, 1993, p. 179. Traducción del alemán del autor de este texto. Véase también M. ManuntA, «T. S. Eliot e F. H. Bradley: la tradizione come surrogato dell'assoluto", Acme, LVI, 2 (2003), pp. 199-218.

[12] E. Cassirer, op. cit., p. 179. 
finita en un sistema superrelacional y unitario de experiencia que armonice todo tipo de diferencia y multiplicidad.

Siguiendo los argumentos de Appearance and Reality sin preocuparse, por el momento, de la paradoja mencionada más arriba, afirmar que «lo Absoluto es sus apariencias» ${ }^{13}$ representa la verdadera perspectiva desde la cual habría que mirar al binomio finito-infinito. Entre la unidad de la realidad en lo Absoluto, en cuanto no debe implicar las contradicciones típicas de las entidades finitas y del pensamiento relacional, y las apariencias, no puede existir ninguna relación: de lo contrario, se establecería otra serie de nexos contradictorios, como en el caso de las cosas y de las categorías finitas a las que Bradley se refiere en la primera parte de Appearance and Reality. Aunque el lenguaje hegeliano implique ciertas asunciones no típicamente bradleyanas, se podría expresar esta idea de la misma manera en que el filósofo alemán trata, en la Ciencia de la Lógica, el infinito malo:

el infinito, puesto contra lo finito en una relación cualitativa de otros el uno frente al otro, tiene que llamarse el falso-infinito [..]. Esta contradicción se presenta en seguida en esto, que lo finito permanece como existencia frente al infinito. Hay por ende dos determinaciones; se dan dos mundos, uno infinito y otro finito, y en su relación el infinito es sólo el término de lo finito, y por lo tanto sólo un infinito determinado, que es él mismo un infinito finito. ${ }^{14}$

A raíz de estas consideraciones debería resultar claro que, al menos desde un punto de vista general, existen elementos comunes entre la doctrina de Spinoza, el idealismo hegeliano y la filosofía de Bradley: la metafísica debe

[13] F.H. Bradley, Appearance and Reality, p. 486.

[14] G.W.F. HegeL, WL (1832), GW21, 127 (traducción de A. Mondolfo y R. Mondolfo, Prólogo de R. Mondolfo, Buenos Aires: Solar, 1993, 2 Vols., Vol. 1, pp. 178-179). Todas las obras de Hegel se citan según el tomo y la página de G.W.F. Hegel, Gesammelte Werke. Im Auftrag der Deutschen Forschungsgemeinschaft hrsg. v. der Rheinisch-Westfälische Akademie der Wissenschaften, unter der Leitung v. O. Pöggeler, Hamburg: Meiner, 1968 ss. Las Lecciones se citan según el tomo y la página de G.W.F. HegeL, Vorlesungen über die Geschichte der Philosophie. Hrsg. v. P. Garniron und W. Jaeschke. 4 Vols. (Vorlesungen. Ausgewählte Nachschriften und Manuskripte, Vols. 6, 7, 8 y 9). Hamburg: Meiner, 1986-1996.

Estoy convencido de que los argumentos de Bradley acerca del bien y de Dios, siempre con las debidas reservas y limitadamente a este contexto concreto, se acercan de manera muy evidente a la perspectiva hegeliana, pero no a la de Spinoza. Véanse F.H. BradLEY, Appearance and Reality, en particular el capítulo "Goodness», pp. 401-454; F.H. Bradley, Ethical Studies, Second Edition Revised, with an Introduction of R. Wollheim, Oxford: Clarendon Press, 1961, en particular el ensayo «Duty for Duty's Sake», pp. 142-159; J.C. KING, «Bradley's 'Duty for Duty's Sake' and Kant's Ethics», Kant-Studien. Philosophische Zeitschrift der Kant-Gesellschaft, Vol. 59, Issue 1-4 (Jan. 1968), pp. 309-317. 
ocuparse de la construcción de un sistema monista en el que se reconozca la prioridad de lo infinito sobre lo finito y debe llegar al conocimiento de la Realidad en oposición a la mera apariencia basándose en el estudio de los primeros principios y de las verdades últimas. El universo debe de comprenderse como un entero, porque «solamente se da una Realidad». ${ }^{15}$ Esto explica, además, por qué es difícil encontrar una investigación sobre la filosofía bradleyana en la que no se reconozca «la oportunidad [..] de una referencia a Spinoza: como una ascendencia, clásica y moderna a la vez, que Bradley vuelve a proponer, según modalidades igualmente [..] severas y rigurosas a las que se encuentran en la celebérrima producción del autor de la Ética». ${ }^{16}$

\section{Appearance and Reality: Las Críticas de Seth Pringle-Pattison}

La presencia de estos elementos es justamente lo que sugiere a Seth PringlePattison la reflexión acerca de dos tendencias conflictivas dentro de la filosofía bradleyana: en primer lugar «la tendencia spinoziana [..., que en el conjunto es predominante»; ${ }^{17}$ en segundo lugar, la tendencia hegeliana. La razón por la que los principios de la filosofía de Spinoza predominan depende en gran parte de las consecuencias de la reforma del hegelismo llevada a cabo por Bradley, quien es consciente de que su planteamiento se convierte en una verdadera «herejía». ${ }^{18}$ A diferencia de Hegel, para quien los momentos de la dialéctica son las mediaciones gracias a las que el espíritu puede reconciliarse consigo mismo en la forma de la $\theta \varepsilon \omega p i \alpha,{ }^{19}$ Bradley renuncia en Appearance and Reality a todo tipo de explicación relacional de la realidad, de manera que el universo, presente solamente en los centros finitos de experiencia, ha de ser unificado por una exigencia racional de no-contradicción, aun cuando su razón permanezca desconocida. ${ }^{20} \mathrm{El}$ principio platónico de que «lo diferente no es lo mismo que

[15] F.H. Bradley, Appearance and Reality, p. 33. Aunque no podemos desarrollarlo en detalle, Candlish resume esta posición con el concepto de consistency-theorem, García con el de Absoluto como totalidad de los hechos. Wollheim habla de los principios de la "imposibilidad de un hecho" y de la "interdependencia entre hechos".

[16] A. Deregibus, loc. cit., XI (1989), p. 34. Traducción del italiano del autor de este texto.

[17] A. Seth Pringle-Pattison, loc. cit., pp. 191-192.

[18] F.H. Bradley, The Principles of Logic, Vol. II, p. 410. Para Bradley, dicha herejía debería salvar la esencia de la doctrina hegeliana. Remito a las conclusiones para la discusión de este punto.

[19] Véanse B. Brinkmann, Idealism Without Limits: Hegel and the Problem of Objectivity, Dordrecht-Heidelberg-London-New York: Springer, 2011; G.W.F. Hegel, Phän., GW9, en particular pp. 422-434 (traducción de M. Jiménez Redondo, Valencia: Pre-Textos 2006, pp. 461473); C. Glimpel, "'Die Darstellung Gottes, wie er in seinem ewigen Wesen ist' - Anmerkungen zum Gottesverständnis in Hegels Wissenschaft der Logik”, en R. Hiltscher-S. Klingner (Hrsg.), Georg Wilhelm Friedrich Hegel, Darmstadt: WBG, 2012, pp. 105-116.

[20] Por esta misma razón algunos críticos han hablado de la necesidad de admitir un acto 
lo uno ni es lo mismo que el ser ${ }^{21}$ se transforma, reinterpretado por Bradley según una acepción particular del lema omnis determinatio est negatio, en un hiato irresoluble entre finito e infinito. Este hiato, fundado en el concepto de transmutación armónica, implica que si fuese posible contemplar el universo sub specie Dei, la derivación de lo finito podría comprenderse de manera lógica y racional; «sin embargo no es posible alcanzar esa perspectiva. Cuando se intente observar desde ese punto de vista, el resultado es, como en el caso de Spinoza, el simple vacío».22

Estas consideraciones dependen de la siguiente observación: puesto que la investigación lógica bradleyana, si ha de ser verídica y sincera, tiene que ser crítica y escéptica, «la fuerza entera de su argumento [..] parece basarse en la ilegítima extensión a la realidad o a la experiencia de una ley que solamente es válida para los conceptos»: 23 el principio de no-contradicción. La tarea de Bradley, por tanto, es tan ambiciosa como la de Hegel, ya que la metafísica no renuncia a la definición del universo, si bien la falta de una respuesta definitiva depende del fracaso del pensamiento relacional (el único del que disponemos los seres finitos). En consecuencia, Seth Prigle-Pattison observa que, a falta de una efectiva correspondencia entre el pensamiento y la Realidad, Bradley abandona el finito en un limbo ontológico en el que lo Absoluto no puede siquiera concebirse, porque todo pensamiento relacional se reduce a ser mero expediente, recurso, compromiso práctico. ${ }^{24} \mathrm{Al}$ carecer de una deducción evidente y coherente del mundo finito (los modos de la causa sui), la doctrina bradleyana postularía una identidad abstracta donde las apariencias han de transmutarse, disolverse y perderse. Como en el caso de Spinoza, por tanto, lo Absoluto no tendría el carácter activo y vivo requerido para poder determinarlo como espíritu. ${ }^{25}$

de fe y, consecuentemente, han relacionado la doctrina bradleyana con la filosofía de Leibniz. Véanse T.S. Eliot, «The Development of Leibniz' Monadism» y «Leibniz' Monads and Bradley's Finite Centres», en T. S. Eliot, op. cit., pp. 177-197 y 198-207; G. STOcK, «Thought and Sensibility in Leibniz, Kant and Bradley», en T. McWalter-G. MacDonald Ross (edited by), op. cit., pp. 104-125.

[21] Platón, Parménides, en Platón, Dialogos, Introducciones, traducciones y notas de Ma.I. Santa Cruz, Á. Vallejo Campos, N. Luis Cordero, 8 Vols., Madrid: Gredos 2007, Vol. v, p. 71.

[22] A. Seth Pringle-Pattison, loc. cit., p. 179.

[23] Ibid.,p. 158.

[24] Véanse F.H. Bradley, Appearance and Reality; F.H. Bradley, Essays on Truth and Reality, Oxford: Clarendon Press 1914, pp. 409-427.

[25] Véase G.W.F. HegeL, VGPh9, 103-105. Al respecto, recuérdese el éxplicit de Appearance and Reality: «se podría justamente concluir esta obra insistiendo en la idea de que la Realidad es espiritual. Podría mencionar una famosa máxima de Hegel, mas no me atrevo a hacerlo sin aportar las debidas explicaciones. Sin embargo, terminaré con algo muy parecido, algo que probablemente se acerca más al mensaje de Hegel. Por fuera del espíritu hay, y no puede haber, ninguna realidad, y cuanto más una cosa es espiritual, tanto más es verdaderamente real» [F.H. Bradley, Appearance and Reality, p. 552]. Para Seth Pringle-Pattison la doctrina de Bradley no se 
El apoyo en los hechos concretos de la experiencia representaría, para Seth Pringle-Pattison, el único recurso para superar esta dificultad, puesto que para Bradley «la identidad solo existe en la diferencia, la unidad en la multiplicidad». ${ }^{26}$ En efecto, la apelación a la experiencia, al feeling, a un nivel experiencial básico, pre-relacional, con el que empieza todo conocimiento y «en el que no hay distinción entre mi conciencia y lo de que estoy consciente», ${ }^{27}$ permite a Bradley dinamizar el mundo de las apariencias garantizando un movimiento constante, de acuerdo con un sistema de grados y jerarquías que se diferenciaría así de la sustancia spinoziana. La doctrina de los grados de verdad y realidad, desarrollada en Appearance and Reality mas vinculada de forma esencial con los principios de la lógica, muestra que este ground en que se da un todo-en-uno y que, a pesar de ser continuamente trascendido, permanece siempre presente, representa la única evidencia para poder concebir lo Absoluto.

Contra el «absurdo ballet de categorías sin vida $»^{28}$ que caracteriza la transcendencia vacía del panteísmo, Bradley desarrollaría así la su segunda tendencia, la más auténtica y perdurable herencia hegeliana: la realización y la revelación de lo infinito en lo finito. La condición de posibilidad de la realización de lo Absoluto consiste en su propio desarrollo según una estructura ordenada de contenidos organizados según el principio de que más verdadero y real es aquel contenido determinado que, en su acto de transcendencia para encontrar su compleción en lo Absoluto, necesita de una integración menor. De hecho, si lo Absoluto es una totalidad armónica omninclusiva, es evidente que la verdad y la realidad de un contenido empírico o del juicio que lo expresa residen en la menor o mayor distancia de la imperfección:

en esta serie el puro Espíritu se hallaría en el extremo más alejado de la naturaleza sin vida. Al aumentar de grado en esta serie, se encontraría más del primer aspecto y menos del segundo [..]. El Espíritu es una unidad de diversos en la que la singularidad de la diferencia deja completamente de existir. ${ }^{29}$

La doctrina de los grados de verdad y realidad implica, sin embargo, otra importante cuestión metafísica: el pensamiento no puede alcanzar ningún tipo de Aufhebung que permita superar las contradicciones de las apariencias y llegar a la Realidad en su plenitud. En este sentido, observa Seth Pringle-Pattison, el

diferenciaría del inmovilismo de la sustancia de Spinoza, donde todo entra para para desaparecer en ella, y de la que nada sale (como en una Löwenhöhle).

[26] A. Seth Pringle-Pattison, loc. cit., p. 159.

[27] F.H. Bradley, Essays on Truth and Reality, p. 159.

[28] F.H. Bradley, The Principles of Logic, Vol. II, p. 591.

[29] F.H. Bradley, Appearance and Reality, p. 498. 
intento de Bradley de complementar a Hegel - o incluso de superarle - consiste en abandonar el planteamiento panlogísta y, consecuentemente, en rechazar la posibilidad de poder dar una «representación de Dios, tal como está en su ser eterno, antes de la creación de la naturaleza y de un espíritu finito». ${ }^{30}$ La filosofía de la inmanencia de Hegel y la reivindicación de la validez del conocimiento cometería el error de "poner el filósofo en el lugar de la divinidad, y de identificar literalmente la historia de la humanidad con el desarrollo de lo Absoluto», ${ }^{31}$ expuesto según los principios de la dialéctica. Oponiéndose al planteamiento supuestamente humano, demasiado humano de Hegel y al ballet de sus categorías, Bradley defiende la idea de que «solamente se da una Realidad, y su naturaleza puede definirse como experiencia». ${ }^{32}$ De esta manera, transforma el idealismo en una filosofía de lo finito que, puesta la incognoscibilidad de lo Absoluto, ni siquiera puede aspirar a la verdad, un hecho que, para Seth Pringle-Pattison, «expresa una disposición filosófica esencialmente brahmánica».33

\section{CONCLUSIONES: ¿UN ESPINOSISTA HEGELIANO?}

Los argumentos que se han ido exponiendo en el presente artículo discuten desde un punto de vista teorético la presencia de dos tendencias fundamentales dentro de la filosofía bradleyana. El análisis de Seth Pringle-Pattison resulta ciertamente interesante para la comprensión de algunos elementos críticos de Appearance and Reality y, de forma indirecta, de todo el sistema metafísico tal y como se desarrolla en las otras importantes obras de su autor, porque Spinoza y Hegel constituyen indudablemente una referencia importante para Bradley. Se ha visto, además, que las consideraciones llevadas a cabo en «A New Theory of the Absolute» reciben cierto asenso (directo o indirecto) de parte de otros críticos relevantes. A raíz de los argumentos expuestos en el

[30] G.W.F. Hegel, WL (1832), SW21, 34 (traducción de A. Mondolfo y R. Mondolfo, Vol. 1, p. 66).

[31] A. Seth Pringle-Pattison, loc. cit., p. 202. El autor remite directamente a las críticas llevadas a cabo por Lotze contra el sistema hegeliano. Piénsese también a las palabras de Cassirer: desde la perspectiva hegeliana, «para que la filosofía sea la conciencia de sí mismo verdadera y adecuada del espíritu, no basta con que establezca unas cuantas reglas escuetas y abstractas acerca del funcionamiento general del espíritu. Para ello tiene, por el contrario, que encuadrar verdaderamente dentro de sí todo el trabajo espiritual creador, la totalidad del "espíritu objetivo", tal como se representa en la religión y en el arte, en la moral y en el derecho, en la ciencia y en el Estado: no puede limitarse a señalar las condiciones de la cultura espiritual, sino que tiene que aprisionar y conservar dentro de sí todo el contenido, bajo la forma del pensamiento» [E. CASSIRER, El problema del conocimiento en la filosofía y en la ciencia modernas, edición de W. Roces, 4 Vols., México DF: FCE, 2003, Vol. 3: Los sistemas postkantianos, p. 437].

[32] F.H. Bradley, Appearance and Reality, p. 455. Cursiva mía.

[33] A. Seth Pringle-Pattison, loc. cit., p. 201. 
ensayo, sin embargo, estoy convencido de que la lectura propuesta por Seth Pringle-Pattison no puede considerarse completa: no solamente porque sus conclusiones niegan el esfuerzo teorético de Bradley (comparando de manera errónea su metafísica a una suerte de misticismo brahmánico) ${ }^{34}$ sino también por la forma de presentar el idealismo de Appearance and Reality en cuanto a su relación con Spinoza y Hegel.

Efectivamente, el sistema filosófico de la Ética plantea el mismo problema metafísico de Bradley ya desde su primera parte, en las definiciones de Dios, de los atributos y de los modos. La relación entre finito e infinito asume la forma del esse in alio y transforma el concepto de Dios en un mundo de modos (Deus sive natura). Sin embargo, la identidad entre Dios, naturaleza y sustancia es justamente lo que garantiza, en la inmanencia, la solución de la correspondencia entre unidad y multiplicidad: de hecho, el principio de máxima armonía para lo finito consiste en su propia auto-limitación como condición del reconocimiento de Dios en nosotros y de nosotros en Dios a través del concepto de beatitud como virtud suprema. En palabras de Spinoza:

la suprema virtud del alma consiste en conocer a Dios [..], o sea, entender las cosas según el tercer género de conocimiento [..]. De esta suerte, quien conoce las cosas según dicho género pasa a la suprema perfección humana, y, por consiguiente, resulta afectado por una alegría suprema [..]; por ende [..], de ese género de conocimiento nace el mayor contento posible. ${ }^{35}$

En este mismo sentido:

la felicidad no es un premio que se otorga a la virtud, sino que es la virtud misma [..]. La felicidad consiste en el amor hacia Dios [..], y este amor brota del tercer género de conocimiento [..]; por ello, dicho amor [..] debe referirse al alma en cuanto que obra, y, por ende [..], es la virtud misma. ${ }^{36}$

Por tanto, cabe subrayar por lo menos la presencia de una diferencia fundamental entre la doctrina de Spinoza y la filosofía Bradleyana - algo en que Seth Pringle-Pattison no hace hincapié. Desde un punto de vista lógico se podría afirmar que el universo de atributos y modos de la Ética es una totalidad colectiva, si con este término se indica la colección de las entidades finitas tomadas en su conjunto ("todo cuanto es, es en Dios, y sin Dios nada puede ser

[34] Véase, por ejemplo, G. Bertolotti, op. cit..

[35] B. SpInoza, Ética demostrada según el orden geométrico, Introducción, traducción y notas de V. Peña, Madrid: Alianza, 2006, pp. 411-412.

[36] Ibid., p. 427. 
ni concebirse»). ${ }^{37}$ Lo Absoluto de Bradley, al contrario, podría definirse como una totalidad distributiva, puesto que resulta ser el único sujeto de todo posible juicio y para concebirlo y componerlo no basta con yuxtaponer cada ente finito y sus cualidades, sino que se necesita una transfiguración. ${ }^{38}$ Con esto, no quiero aseverar que la sustancia de Spinoza no difiera de los modos en cuanto a su esencia (algo demostrado por las propias definiciones), sino sugerir más bien que lo Absoluto spinoziano, o la propia sustancia, constituye la unidad originaria y se identifica con el Ser supremo, Dios, en que estamos y vivimos en cuanto modos, como parte de su propia definición. Para Bradley, en cambio:

identificando lo Absoluto con Dios, no es posible seguir pensando en el Dios de la religión. Intentando separarles, Dios se transforma en un factor finito del Todo [..]. Se podría decir que Dios no es Dios hasta que no es un todo en todo, y que ese Dios que es un todo en todo no es el Dios de la religión. Dios solo es un aspecto de lo Absoluto, y esto significa que solo es una apariencia. ${ }^{39}$

En consecuencia, como es evidente, el Dios de Spinoza - y con él, por tanto, toda su doctrina - «no es lo Absoluto de Bradley, el amor Dei intellectualis spinoziano no es lo Ignoto de la absolutidad bradleyana». ${ }^{40}$

La inexplicable transfiguración necesaria para comprender la revelación de lo infinito en lo finito implica una suerte de movimiento o de mediación y constituye el aspecto más hegeliano de la filosofía bradleyana. Sin embargo, representa a la vez el elemento más crítico respecto al hiper-relacionalismo de Hegel. En cuanto el contenido de la filosofía es la realidad, advierte Hegel en la Fenomenología, la génesis de su desvelamiento ha de hallarse en la conciencia inmediata, o sea, en la particular relación de la propia conciencia con su objeto, en la que la alteridad es aparentemente inmediata e insuperable. He aquí la razón por la que la certeza inmediata es un conocimiento dotado de infinita riqueza pero, a la vez, es la verdad más abstracta y pobre. Independientemente de las contradicciones implicadas por tal punto de partida, que ponen en marcha el vertiginoso movimiento de las figuras de la conciencia que apunta al saber absoluto, este primer momento de la Fenomenología (a pesar de ser el más cercano al concepto de feeling presentado por Bradley) no corresponde en ningún modo a las premisas metafísicas asumidas por el filósofo inglés.

[37] Ibid., p. 62.

[38] Sobre la acepción de relación algébrica, colectiva y distributiva remito a M. MugnAI, Introduzione alla filosofia di Leibniz, Torino: Einaudi, 2001.

[39] F.H. BRAdLEy, Appearance and Reality, pp. 448-449. Cursiva mía.

[40] A. Deregibus, loc. cit., XI (1989), p. 39. 
La conciencia, de hecho, se encuentra para Hegel en una condición elemental y básica:

en ella una cosa es lo que queda puesto como simple e inmediatamente siendo [..], y ello es el objeto; y otra cosa distinta es lo que queda puesto como lo inesencial y mediado, que en esa certeza no es algo en sí, sino que es puro otro, y éste es el yo, es decir, un saber que sólo sabe de ese objeto porque ese objeto es, y que por su parte es un saber que puede ser, pero que podría también no ser. ${ }^{41}$

La oposición entre sujeto y objeto, que aquí se presupone, muestra toda la distancia entre los planteamientos de Hegel y Bradley. Nada existe fuera del dominio de las relaciones que se hallan en las figuras y entre las figuras, cuya esencia, finalmente, consiste en un agregado de notas imperfectas y contradictorias que se resuelven en una síntesis superior (la Aufhebung) que permite reconocerlas como verdaderas cuando se las mire sub specie Dei. De esta manera, como es evidenciado por el mismo Hegel, el término "experiencia" no denota un ámbito extra-relacional, inmediato y meramente sensible, sino que se refiere al propio movimiento dialéctico que «la conciencia ejercita en ella misma, es decir, tanto en su saber como en su objeto, en cuanto en ese movimiento le salta o le brota a ella el nuevo objeto verdadero, es lo que propiamente se llama experiencia». ${ }^{42}$ Así, pues, por mucho que no se pueda negar la influencia del idealismo alemán (de Hegel especialmente) sobre la producción filosófica de Bradley, su doctrina no puede asimilarse al planteamiento dialéctico del propio Hegel, porque la idealidad defendida en Appearance and Reality no implica una identificación con la realidad ni la coincidencia de lógica, metafísica y ontología. Al contrario, para Bradley la propia idealidad «ha de ser superada en la absolutidad de la "realidad", la cual, cuanto más se reconoce y declara tal gracias a su propia profundización y unificación, [..] tanto más adquiere "perfección" unitaria y totalizante» ${ }^{43}$, mas no relacional y, por tanto, no del todo racional.

En conclusión: puesto que ningún grado de conocimiento es capaz de llegar a la Realidad, el salto inexplicable en que consiste la transmutación de las apariencias en el orden supra-relacional que caracteriza lo Absoluto bradleyano implica una diferencia esencial entre la realidad y sus apariencias, entre lo infinito y lo finito, Dios y sus modos. Por estos motivos no puedo compartir integramente la interpretación de Seth Pringle-Pattison ya que no considero que las tendencias espinosista y hegeliana de Bradley sean suficientes para de-

[41] G.W.F. Hegel, Phän., GW9, 64 (traducción de M. Jiménez Redondo, p. 199).

[42] Ibid., 60 (traducción de M. Jiménez Redondo, p. 191).

[43] A. Deregibus, loc. cit., XI (1989), p. 37. 
finir y comprender de forma correcta su sistema metafísico: Bradley no es ni espinosista ni hegeliano.

\section{REFERENCIAS BIBLIOGRÁFICAS}

BEDELL, G., «Bradley's Monistic Idealism», The Thomist: A Speculative Quarterly Review, 34, 4 (Oct 1, 1970), pp. 568-583.

BERTOLOTTI, G., Le stagioni dell'assoluto: saggio su Bradley, Firenze: La Nuova Italia, 1995.

BRADLEY, F.H., Appearance and Reality: A Metaphysical Essay, Elibron Classics, 2005.

BRADLEY, F.H., Ethical Studies, Second Edition Revised, with an Introduction of R. Wollheim, Oxford: Clarendon Press, 1961.

BRADLEY, F.H., Essays on Truth and Reality, Oxford: Clarendon Press, 1914.

BRADLEY, F.H., The Principles of Logic, Second Edition revised with Commentary and Terminal Essays, edited by H. Milford, 2 Vols., London: Oxford University Press, 1950.

BRINKMANN, B., Idealism Without Limits: Hegel and the Problem of Objectivity, Dordrecht-Heidelberg-London-New York: Springer, 2011.

CANDLISH, S., «The Truth About F.H. Bradley», Mind New Series, Vol. 98, No. 391 (Jul., 1989), pp. 331-348.

CARBÓ I RIBUGENT, M., «Henry Crabb Robinson i el context filosòfic de la primera importació de Kant a Anglaterra», Anuari de la Societat Catalana de Filosofia, XIX (2008), pp. 35-56.

CARDANI, M., «Por un puñado de... táleros. Existencia y contenido empírico en F. H. Bradley», Comprendre: Revista catalana de filosofia, Vol. 16, Núm. 2 (2014), pp. 51-70.

CASSIRER, E., El problema del conocimiento en la filosofía y en la ciencia modernas, edición de W. Roces, 4 Vols., México DF: FCE, 2003, Vol. 3: Los sistemas postkantianos.

CASSIRER, E., Erkenntnis, Begriff, Kultur, herausgegeben, eingeleitet sowie mit Anmerkungen und Registern versehen von R.A. Bast, Hamburg: Meiner, 1993.

DEREGIBUS, A., «Bradley e Spinoza. L’iper-spinozismo di F.H. Bradley», Giornale di Metafisica Nuova Serie, x (1988), pp. 339-392 у xi (1989), pp. 3-40.

ELIOT, T.S., Knowledge and Experience in the Philosophy of F. H. Bradley, London: Faber \& Faber, 1964. 
GARCÍA, P.S., «Sobre la noción de absoluto en F.H. Bradley», Thémata. Revista de filosofía, 18 (1997), pp. 189-196.

GLIMPEL, C., “Die Darstellung Gottes, wie er in seinem ewigen Wesen ist” Anmerkungen zum Gottesverständnis in Hegels Wissenschaft der Logik», en HILTSCHER, R.-KLINGNER, S. (Hrsg.), Georg Wilhelm Friedrich Hegel, Darmstadt: WBG, 2012, pp. 105-116.

HEGEL, G.W.F., Phänomenologie des Geistes, Phän, en HEGEL, G.W.F., Gesammelte Werke. Im Auftrag der Deutschen Forschungsgemeinschaft hrsg. v. der RheinischWestfälische Akademie der Wissenschaften, unter der Leitung v. O. Pöggeler, Band 9, Hamburg: Meiner, 1980 (traducción de M. Jiménez Redondo, Valencia: Pre-Textos 2006).

HEGEL, G.W.F., Vorlesungen über die Geschichte der Philosophie, VGPh, en HEGEL, G.W.F., Vorlesungen. Ausgewählte Nachschriften und Manuskripte, Hrsg. v. P. Garniron und W. Jaeschke, Band 9, Hamburg: Meiner, 1986.

HEGEL, G.W.F., Wissenschaft der Logik, WL, en HEGEL, G.W.F., Gesammelte Werke. Im Auftrag der Deutschen Forschungsgemeinschaft hrsg. v. der RheinischWestfälische Akademie der Wissenschaften, unter der Leitung v. O. Pöggeler, Band 21, Hamburg: Meiner, 1985 (traducción de A. Mondolfo y R. Mondolfo, Prólogo de R. Mondolfo, Buenos Aires: Solar, 1993, 2 Vols.).

HØFFDING, H., Modern Philosophers, London: MacMillan and Co., 1915.

KING, J.C., «Bradley's 'Duty for Duty's Sake' and Kant's Ethics», Kant-Studien. Philosophische Zeitschrift der Kant-Gesellschaft, Vol. 59, Issue 1-4 (Jan. 1968), pp. 309-317.

MANDER, W.J., British Idealism. A History, Oxford: Oxford University Press 2011.

MANUNTA, M., «T. S. Eliot e F. H. Bradley: la tradizione come surrogato dell'assoluto», Acme, LVI, 2 (2003), pp. 199-218.

METZ, R., A Hundred Years of British Philosophy, London: George Allen \& Unwin ltd., 1938.

MICHELI, G., «The early reception of Kant's Thought in England 1785-1805», en MCWALTER, T.-MACDONALD ROSS, G. (edited by), Kant and his influence, London: Thoemmes Press, 1990, pp. 202-314.

MUGNAI, M., Introduzione alla filosofia di Leibniz, Torino: Einaudi, 2001.

MUIRHEAD, J.H., «How Hegel came to England», Mind New Series, Vol. 36, No. 144 (Oct., 1927), pp. 423-447.

NEWLANDS, S., «More Recent Idealist Readings of Spinoza», Philosophy Compass, 6/2 (2011), pp. 109-119.

PLATÓN, Parménides, en PLATÓN, Dialogos, Introducciones, traducciones y notas de Ma.I. Santa Cruz-Á. Vallejo Campos-N. Luis Cordero, 8 Vols., Madrid: Gredos, 
2007, Vol. v, pp. 25-132.

PUCELLE, J., L'idéalisme en Angleterre de Coleridge à Bradley, Neuchatel: La Bacconière, 1955.

SETH, A.-HALDANE, R.B. (edited by), Essays in Philosphical Criticism, with a Preface of E. Caird, New York: Burt Franklin, 1971.

SETH PRINGLE-PATTISON, A., "A New Theory of the Absolute. Mr. Bradley's Appearance and Reality», en SETH PRINGLE-PATTISON, A., Man's Place in Cosmos and Other Essays, Edinburgh-London: Blackwood and Sons, 1897, pp. 129-225.

SPINOZA, B., Ética demostrada según el orden geométrico, Introducción, traducción y notas de V. Peña, Madrid: Alianza, 2006.

STOCK. G., «Thought and Sensibility in Leibniz, Kant and Bradley», en MCWALTER, T.-MACDONALD ROSS, G. (edited by), Kant and his influence, London: Thoemmes Press, 1990, pp. 104-125.

TRUGLIA, M., F.H. Bradley e la genesi della filosofia analitica. Contributi per una definizione, Milano: Unicopli, 2007.

WATTS CUNNINGHAM, G., The Idealistic Argument in Recent British and American Philosophy, New York: Books for Libraries Press, 1967.

WELLEK, R., Immanuel Kant in England, 1793-1838, Princeton University Press, 1931.

WOLlHEIM, R., F.H. Bradley, Harmondsworth-Baltimore-Ringwood: Penguin, 1969.

Michele Cardani is a Ph.D. candidate in Contemporary Philosophy and Classical Studies at the Department of Theoretical and Practical Philosophy at the University of Barcelona.

RESEARCH INTERESTS:

British Idealism; Contemporary Renovation of Transcendentalism; German Idealism; History of Idealism; History of Modern Philosophy; Kantian and Neo-Kantian Movements. 
AdDress:

Departament de Filosofia Teorètica i Pràctica

Universitat de Barcelona, Facultat de Filosofia

Carrer Montalegre, 6-8, 08001, Barcelona.

\section{RECENT PUBLICATIONS:}

CARDANI, M., «Vuelta a la realidad: nuevo realismo contra Kant», en NAvARro Cordón, J.m.-Orden Jiménez, R.v.-Rovira, R. (eds.) Nuevas perspectivas sobre la filosofía de Kant, Madrid: Escolar y Mayo, 2016, pp. 57-66.

CARDAni, M., «Por un puñado de... táleros. Existencia y contenido empírico en F. H. Bradley», Comprendre: Revista catalana de filosofia, Vol. 16, Núm. 2 (2014), pp. 51-70.

CARDANi, M., «Per una ricostruzione storico-teoretica dell'idealismo britannico», Humanitas, 69, 1/2014, pp. 172-181.

CARDAni, M., «Nullità della dialettica hegeliana. L'eresia ortodossa di F.H. Bradley», en Pozzoni, I. (a cura di), Frammenti di filosofia contemporanea I, Villasanta: Limina Mentis, 2012, pp. 13-34.

Cardani, M., "La copia in gesso di un uomo vivente. Perché, di nuovo, Kant?», Humanitas, forthcoming.

Cardani, M.-TAmborini, M., «Data-Phenomena: Quid Juris?», Zeitschrift für philosophische Forschung, forthcoming.

CARdani, M.-TAmborini, M., «Italian New Realism and Trascendental Philosophy: A Critical Account», Philosophy Today, forthcoming. 